\title{
De novo POGZ mutations in sporadic autism disrupt the DNA-binding activity of POGZ
}

\author{
Kensuke Matsumura', Takanobu Nakazawa², Kazuki Nagayasu², Nanaka Gotoda-Nishimura', Atsushi Kasai', \\ Atsuko Hayata-Takano ${ }^{1}$, Norihito Shintani ${ }^{1}$, Hidenaga Yamamori ${ }^{3}$, Yuka Yasuda ${ }^{3}$, Ryota Hashimoto ${ }^{3,4}$ \\ and Hitoshi Hashimoto ${ }^{1,2,4}$
}

\begin{abstract}
Background: A spontaneous de novo mutation is a new mutation appeared in a child that neither the parent carries. Recent studies suggest that recurrent de novo loss-of-function mutations identified in patients with sporadic autism spectrum disorder (ASD) play a key role in the etiology of the disorder. POGZ is one of the most recurrently mutated genes in ASD patients. Our laboratory and other groups have recently found that POGZ has at least 18 independent de novo possible loss-of-function mutations. Despite the apparent importance, these mutations have never previously been assessed via functional analysis.

Methods: Using wild-type, the Q1042R-mutated, and R1008X-mutated POGZ, we performed DNA-binding experiments for proteins that used the CENP-B box sequence in vitro. Data were statistically analyzed by one-way ANOVA followed by Tukey-Kramer post hoc tests.

Results: This study reveals that ASD-associated de novo mutations (Q1042R and R1008X) in the POGZ disrupt its DNA-binding activity.

Conclusions: Here, we report the first functional characterization of de novo POGZ mutations identified in sporadic ASD cases. These findings provide important insights into the cellular basis of ASD.
\end{abstract}

Keywords: Autism spectrum disorder, Recurrent mutation, De novo mutation, POGZ, DNA-binding activity

\section{Background}

The genetic etiology of autism spectrum disorder (ASD) remains poorly understood. A spontaneous de novo mutation is a new mutation appeared in a child that neither the parent carries. Recent next-generation sequencing studies have demonstrated that de novo mutations greatly contribute to the risk of ASD and often produce large effects [1-5]. In particular, genes with highly recurrent de novo possible loss-of-function mutations play key roles in the etiology of this disorder. De novo mutations in multiple $(\geq 3)$ unrelated patients have been identified in several such high-confidence ASD risk genes,

\footnotetext{
* Correspondence: takanobunakazawa-tky@umin.ac.jp

${ }_{2}$ iPS Cell-Based Research Project on Brain Neuropharmacology and

Toxicology, Graduate School of Pharmaceutical Sciences, Osaka University,

1-6 Yamadaoka, Suita, Osaka 565-0871, Japan

Full list of author information is available at the end of the article
}

including CHD8, ARID1B, SYNGAP1, DYRK1A, SCN2A, ANK2, ADNP, DSCAM, CHD2, KDM5B, SUV42OH1, GRIN2B, ASH1L, and POGZ [5]. Among these 14 genes, POGZ is one of the most recurrently mutated genes in ASD patients $[4,5]$. Our laboratory and other groups have recently found that POGZ has at least 18 independent de novo possible loss-of-function mutations (Fig. 1, upper) [4-8]. Therefore, de novo mutations in POGZ can be strongly associated with ASD risk; however this association requires experimental validation. Despite the apparent importance, these mutations have never previously been assessed via functional analysis. Here, we report that ASD-associated de novo mutations in the POGZ disrupt the DNA-binding activity of POGZ. These findings provide insight into the cellular basis of ASD. In addition, de novo POGZ mutations are frequently found also in patients with intellectual 

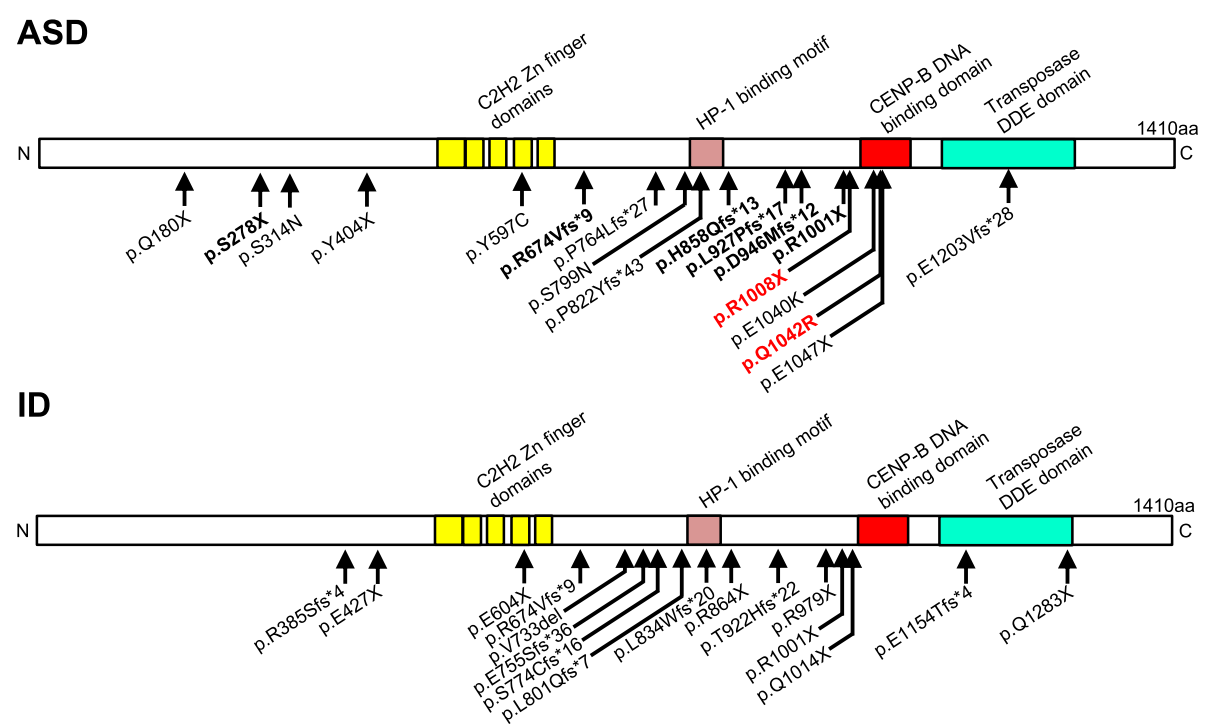

Fig. 1 Schematic structure of POGZ and its putative functional domains. The ASD-(upper) and ID- (lower) associated de novo mutations are indicated below the protein. Bold mutations, common de novo mutations between ASD and ID. Note that the R1001X mutation was found in ID and ASD/ID patients. X, nonsense; del, deletion; fs, frameshift; * , premature stop codon

disability (ID) [7, 9-11] (Fig. 1, lower). Our current findings may also help to understand the molecular etiology of ID.

\section{Methods}

\section{Cell culture and DNA transfection}

Human POGZ cDNA was purchased from DNAFORM (clone ID: 30745658, Kanagawa, Japan), amplified via PCR, and subcloned into a pcDNA-6Myc expression vector. Single amino acid mutants of POGZ were generated using a KOD mutagenesis kit (Toyobo, Osaka, Japan) in accordance with the manufacturer's instructions. HEK293T cells were cultured in Dulbecco's Modified Eagle Medium supplemented with $10 \%$ fetal bovine serum and were transfected using TransIT Transfection Reagent (Takara, Ohtsu, Japan). Two days later, cells were harvested and lysed with TNE buffer (50 mM Tris- $\mathrm{HCl}$ (pH 7.5); $100 \mathrm{mM} \mathrm{NaCl} ; 5$ mM EDTA; $0.1 \%$ (w/v) NP-40) for the DNA-binding assay [12].

\section{Antibodies}

Antibodies used in this study were obtained commercially and included antibodies against POGZ (Sigma-Aldrich, MO, USA), Tuj1 (Covance, CA, USA), GAPDH (Millipore, MA, USA), Histone H3 (Cell Signaling, MA, USA), and Myc (9E10) (Santa Cruz, CA, USA).

\section{Cortical neuronal cultures and neuron immunocytochemistry}

Cortical cultures were prepared from E16.5 embryonic mouse cortex in minimum essential medium with B27 supplement and $5 \%$ fetal bovine serum and plated on glass coverslips coated with poly-L-lysine, as previously described [13]. Neuron immunocytochemistry (at 7 days in vitro) was performed as previously described [13].

\section{Preparation of cytosolic and nuclear fractions}

The preparation of cytosolic and nuclear fractions from dissociated cortical neurons (at 7 days in vitro) was performed using a Cytoplasmic \& Nuclear Protein Extraction Kit (101Bio, CA, USA) in accordance with the manufacturer's instructions.

\section{Immunoblotting}

Bead-bound proteins and lysates were resolved by SDSPAGE and transferred to polyvinylidene difluoride membranes. Subsequently, these membranes were probed with the indicated antibodies. Data acquisition and analysis were performed using an LAS 4000 image analyzer (GE Healthcare, NJ, USA).

\section{Assay for POGZ-CENPB-DB domain binding}

HEK293T cell lysates expressing virtually equivalent levels of Myc-tagged wild-type or mutant POGZ were prepared. These lysates were mixed with $10 \mu \mathrm{g}$ of a 3'-biotinylated DNA fragment carrying the CENP-B box sequence [14] and with NeutrAvidin beads ( $30 \mu \mathrm{l}, 50 \%$ slurry, Thermo Scientific, MA, USA) for $4 \mathrm{~h}$ at $4{ }^{\circ} \mathrm{C}$. After incubation, the beads were collected and washed 4 times with TNE buffer. For quantification, the precipitated POGZs were normalized to each POGZ level in total lysates. The background level was set to the band intensity for the precipitated R1008X POGZ mutant. The levels of precipitated POGZs were normalized to each POGZ level in total lysates. Data 
were statistically analyzed by one-way ANOVA followed by Tukey-Kramer post hoc tests. Differences were considered significant if $p<0.05$.

\section{Results}

POGZ encodes a heterochromatin protein $1 \alpha$-binding protein that contains a zinc-finger cluster, an HP1binding motif, a centromere protein-B-like DNAbinding (CENPB-DB) domain, and a transposase-derived DDE domain [15]. Thus, it has been suggested that POGZ functions as a chromatin regulator [15]. In accordance with this hypothesis, we found that POGZ was localized to the nucleus in neurons (Fig. 2a); however, the function of POGZ in the central nervous system is unknown. We recently discovered a Q1042R amino acid substitution within the CENPB-DB domain in sporadic ASD cases (Fig. 1, upper) [8]. Given that the CENPB-DB domain is likely to be involved in CENP-B box sequence-specific DNA-binding [14], this substitution may affect the DNA-binding activity of POGZ. To examine this possibility, we performed DNA-binding experiments for proteins that used the CENP-B box sequence (Fig. 2b) [14]. We prepared HEK293T cell lysates expressing virtually equivalent levels of Myc-tagged wildtype, Q1042R-mutated, and R1008X-mutated POGZ (Fig. 2b, left, lower). These lysates were mixed with the DNA fragment carrying the CENP-B box sequence. The levels of precipitated POGZs were normalized to each POGZ level in total lysates (Fig. 2b, right). We found that wild-type POGZ co-precipitated well with the DNA fragment carrying the CENP-B box sequence, a clear indication of binding between wild-type POGZ and the CENP-B box sequence (Fig. 2b). Interestingly, the Q1042R mutation was associated with a reduction of approximately $60 \%$ in DNA-binding, suggesting that Q1042 is important for the DNA-binding activity of POGZ (Fig. 2b). We also examined the DNA-binding activity of POGZ carrying the ASD-associated R1008X de novo mutation; this mutation results in a truncated protein that lacks the entire CENPB-DB domain. We found that POGZ with this mutation did not co-precipitate with the DNA fragment (Fig. 2b), indicating the importance of the CENPB-DB domain to the DNA-binding activity of POGZ.

\section{Discussion}

Recently, POGZ is found to be important for normal learning in a habituation paradigm in Drosophila [7]; however, the significance of disease-associated de novo mutations remains unclear. Here, we report the first functional characterization of de novo POGZ mutations identified in sporadic ASD cases. Our results indicate that ASD-associated de novo mutations disrupt the DNA-binding activity of POGZ, an effect likely to result
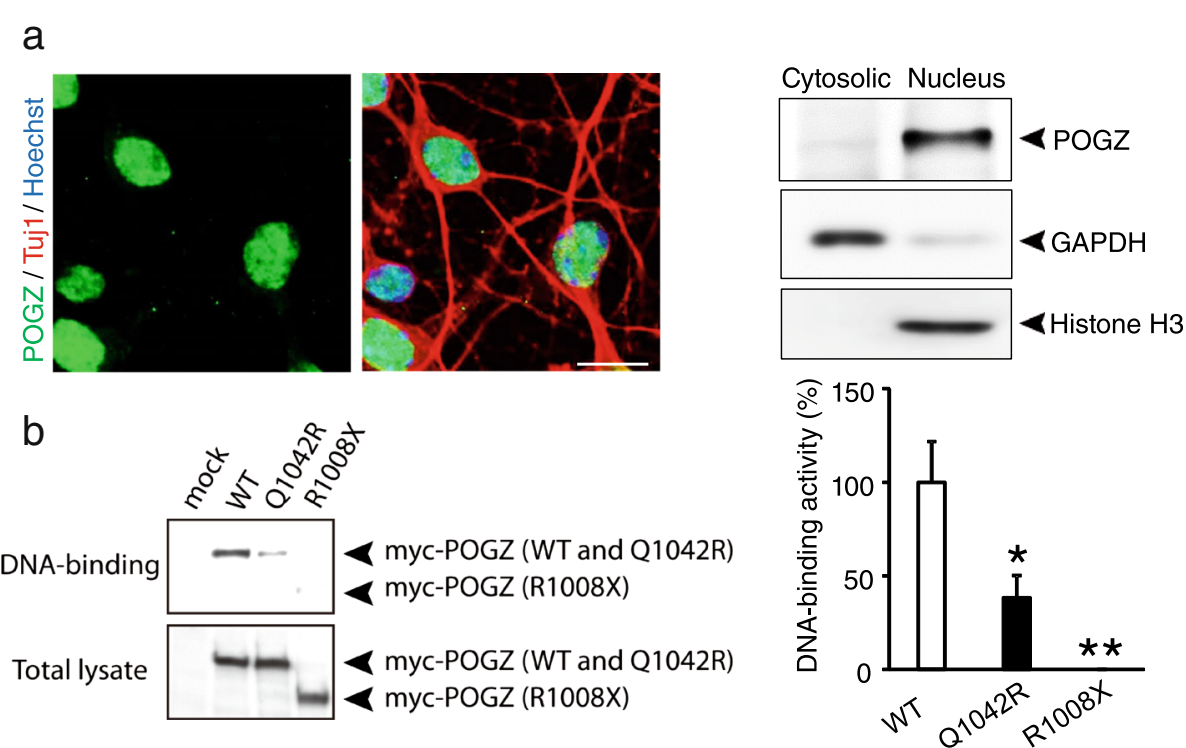

Fig. 2 ASD-associated de novo R1008X and Q1042R mutations disrupt the DNA-binding activity of POGZ. a POGZ is localized to the nucleus in dissociated embryonic cortical neurons (7 days in vitro). left, Double immunostaining for POGZ (green) and a neuron marker, Tuj1 (red). Nuclei were stained with Hoechst 33258 (blue). Scale bar, $10 \mu \mathrm{m}$. right, Biochemical preparation of the cytosolic and nuclear fractions from dissociated neurons. Equal quantities of protein were loaded into individual lanes and probed with antibodies against POGZ, GAPDH (a cytosolic marker), and Histone H3 (a nuclear marker). b Wild-type (WT) but not R1008X POGZ binds a CENP-B box sequence. left, Representative western blots. right, Quantification of DNA-binding activity. Notably, the Q1042R mutation led to a reduction of approximately $60 \%$ in DNA binding (each $n=6$, ${ }^{*} p<0.05,{ }^{* *} p<0.01$, one-way ANOVA followed by Tukey-Kramer post hoc tests (vs. WT)). The averaged WT value was set to $100 \%$. Data are expressed as the means \pm SEM 
in the perturbation of chromatin function and the neuronal transcription network. Given that chromatin regulation plays an essential role in gene expression and cellular function, the disruption of chromatin-related mechanisms causes pathological effects on brain function $[2,16]$. Importantly, 8 out of 14 recurrently mutated high-confidence ASD risk gene products, including POGZ, ADNP, ARID1B, CHD2, KDM5B, SUV420H1, ASH1L, and CHD8, are likely to be chromatin regulators [5]; this finding indicates the critical involvement of chromatin regulation and function in the etiology of ASD $[2,16]$. Interestingly, it has been suggested that POGZ cooperatively regulates chromatin structure and gene expression during human neurodevelopment in combination with a chromatin modifier CHD8, which harbors the largest number of loss-of-function mutations in sporadic ASD [5, 17-19]. Taken together, these findings indicate that both POGZ and CHD 8 may be strongly associated with ASD risk; however the way in which these disruptive de novo mutations of chromatin regulators are involved in ASD risk remains unclear [17-19]. It is important to identify the transcriptional targets of POGZ and CHD8 as well as the biological significance of the disruptive de novo mutations.

Many genes are shown to be associated with ID as well as ASD. Recent studies show that candidate ID- and ASDassociated genes are likely to be largely overlapping [20]. As expected, in addition to ASD [4-8], de novo POGZ mutations are frequently found also in patients with intellectual disability [7, 9-11]. Patients with POGZ mutations show borderline-moderate ID [7] and lower IQ score [8]. Therefore, identification of the significance of diseaseassociated de novo mutations in the POGZ may unravel the common neural systems associated with ASD and ID.

\section{Conclusions}

Our current results, which indicate that de novo mutations in POGZ impair the DNA-binding activity of POGZ, significantly contribute to understanding the molecular link between chromatin remodeling and ASD. Further analysis of the function of de novo mutations in chromatin regulators will provide important clues to the molecular pathophysiology of ASD.

\section{Abbreviations \\ ASD: autism spectrum disorder; CENPB-DB: centromere protein-B-like DNA binding.}

\section{Competing interests}

The authors declare that they have no competing interests.

\section{Authors' contributions}

$\mathrm{KM}, \mathrm{TN}, \mathrm{RH}$, and $\mathrm{HH}$ designed the study. KM, TN, KN, NGN, AK, AHT, and NS, performed biochemical analysis. All authors interpreted the results. TN and $\mathrm{HH}$ wrote the manuscript. All authors participated in the critical revision of the manuscript for assessing important intellectual content. All authors read and approved the manuscript.

\section{Acknowledgements}

This work was supported by Grants-in-Aid for Scientific Research (B) (15H04645) (T.N.), (B) (26293020) (H.H.), (C) (15 K09865) (Y.Y.), a Grant-in-Aid for Challenging Exploratory Research (26670122) (H.H.), (15 K14963) (T.N.), and a Grant-in-Aid for the Program for Advancing Strategic International Networks to Accelerate the Circulation of Talented Researchers (S2603) (H.H.) from the Japan Society for the Promotion of Science; Grants-in-Aid for Scientific Research on Innovative Areas (15H01288 (H.H.) and 15H01286 (T.N.)) from The Ministry of Education, Culture, Sports, Science, and Technology, Japan; and research grants from the Uehara Memorial Foundation, Japan (R.H.), the Program for Creating Future Wisdom, Osaka University (selected in 2014; T.N. and R.H.), and the Takeda Science

Foundation (T.N.).

\section{Author details}

${ }^{1}$ Laboratory of Molecular Neuropharmacology, Graduate School of Pharmaceutical Sciences, Osaka University, 1-6 Yamadaoka, Suita, Osaka 565-0871, Japan. ${ }^{2}$ iPS Cell-Based Research Project on Brain

Neuropharmacology and Toxicology, Graduate School of Pharmaceutical Sciences, Osaka University, 1-6 Yamadaoka, Suita, Osaka 565-0871, Japan. ${ }^{3}$ Department of Psychiatry, Osaka University Graduate School of Medicine, D3, 2-2, Yamadaoka, Suita, Osaka 565-0871, Japan. ${ }^{4}$ Molecular Research Center for Children's Mental Development, United Graduate School of Child Development, Osaka University, Kanazawa University, Hamamatsu University School of Medicine, Chiba University and University of Fukui, 2-2,

Yamadaoka, Suita, Osaka 565-0871, Japan.

Received: 11 March 2016 Accepted: 13 April 2016

Published online: 21 April 2016

\section{References}

1. O'Roak BJ, Deriziotis P, Lee C, Vives L, Schwartz JJ, Giriajan S, et al. Exome sequencing in sporadic autism spectrum disorders identifies severe de novo mutations. Nat Genet. 2011:43:585-9.

2. De Rubeis $\mathrm{S}$, He X, Goldberg AP, Poultney CS, Samocha K, Cicek AE, et al. Synaptic, transcriptional and chromatin genes disrupted in autism. Nature. 2014:515:209-15.

3. Iossifov I, O'Roak BJ, Sanders SJ, Ronemus M, Krumm N, Levy D, et al. The contribution of de novo coding mutations to autism spectrum disorder. Nature. 2014:515:216-21.

4. Krumm N, O'Roak BJ, Shendure J, Eichler EE. A de novo convergence of autism genetics and molecular neuroscience. Trends Neurosci. 2014;37:95-105.

5. Sanders SJ, He X, Willsey AJ, Ercan-Sencicek AG, Samocha KE, Cicek AE, et al. Insights into autism spectrum disorder genomic architecture and biology from 71 risk loci. Neuron. 2015:87:1215-33.

6. Fukai R, Hiraki $Y$, Yofune $H$, Tsurusaki $Y$, Nakashima M, Saitsu H, et al. A case of autism spectrum disorder arising from a de novo missense mutation in POGZ. J Hum Genet. 2015;60:277-9,

7. Stessman HAF, Willemsen MH, Fenckova M, Penn O, Hoischen A, Xiong B, et al. Disruption of POGZ is associated with intellectual disability and autism spectrum disorders. Am J Hum Genet. 2016;98:541-52.

8. Hashimoto R, Nakazawa T, Tsurusaki Y, Yasuda Y, Nagayasu K, Matsumura K, et al. Whole-exome sequencing and neurite outgrowth analysis in autism spectrum disorder. J Hum Genet. 2016;61:199-206.

9. Gilissen $C$, Hehir-Kwa JY, Thung DT, van de Vorst M, van Bon BWM, Willemsen $\mathrm{MH}$, et al. Genome sequencing identifies major cause of severe intellectual disability. Nature. 2014;511:344-7.

10. Tan B, Zou Y, Zhang Y, Zhang R, Ou J, Shen Y, et al. A novel de novo POGZ mutation in a patient with intellectual disability. J Hum Genet. 2016. doi:10. 1038/jhg.2015.156

11. White J, Beck CR, Harel T, Posey JE, Jhangiani SN, Tang S, et al. POGZ truncating alleles cause syndromic intellectual disability. Genome Med. 2016;8:3.

12. Nakazawa T, Komai S, Watabe AM, Kiyama Y, Fukaya M, Arima-Yoshida F, et al. NR2B tyrosine phosphorylation modulates fear learning as well as amygdaloid synaptic plasticity. EMBO J. 2006;25:2867-77.

13. Nakazawa T, Kuriu T, Tezuka T, Umemori H, Okabe S, Yamamoto T. Regulation of dendritic spine morphology by an NMDA receptor-associated Rho GTPase-activating protein, p250GAP. J Neurochem. 2008;105:1384-93.

14. Masumoto H, Masukata H, Muro Y, Nozaki N, Okazaki T. A human centromere antigen (CENP-B) interacts with a short specific sequence in alphoid DNA, a human centromeric satellite. J Cell Biol. 1989;109:1963-73. 
15. Nozawa R, Nagao K, Masuda HT, Iwasaki O, Hirota T, Nozaki, et al. Human POGZ modulates dissociation of HP1alpha from mitotic chromosome arms through Aurora B activation. Nat Cell Biol. 2010;12:719-27.

16. Suliman R, Ben-David E, Shifman S. Chromatin regulators, phenotypic robustness, and autism risk. Front Genet. 2014;5:81.

17. Cotney J, Muhle RA, Sanders SJ, Liu L, Willsey AJ, Niu W, et al. The autismassociated chromatin modifier CHD8 regulates other autism risk genes during human neurodevelopment. Nat Commun. 2015;6:6404.

18. Willsey AJ, Sanders SJ, Li M, Dong S, Tebbenkamp AT, Muhle RA, et al. Coexpression networks implicate human midfetal deep cortical projection neurons in the pathogenesis of autism. Cell. 2013;155:997-1007.

19. Bourgeron T. From the genetic architecture to synaptic plasticity in autism spectrum disorder. Nat Rev Neurosci. 2015;16:551-63.

20. Srivastava AK, Schwartz CE. Intellectual disability and autism spectrum disorders: causal genes and molecular mechanisms. Neurosci Biobehav Rev. 2014;46:161-74.

Submit your next manuscript to BioMed Central and we will help you at every step:

- We accept pre-submission inquiries

- Our selector tool helps you to find the most relevant journal

- We provide round the clock customer support

- Convenient online submission

- Thorough peer review

- Inclusion in PubMed and all major indexing services

- Maximum visibility for your research

Submit your manuscript at www.biomedcentral.com/submit
Biomed Central 